\section{Shifting constant could shake laws of nature}

$\Gamma$ o rom the speed of light to the charge on an electron, the fundamental constants of physics had been assumed to be immutable. But that comfortable assumption is being challenged.

The latest in a series of experiments to question this view suggests that over the past 12 billion years, the ratio of the mass of a proton to that of an electron may have decreased. The result has left physicists curious, sceptical, and more than a little stumped.

Protons are about 1,836 times heavier than electrons. The exact mass ratio can be calculated by observing how a cloud of hydrogen molecules (each composed of two protons and two electrons) absorbs ultraviolet laser light. Wim Ubachs of the Free University in Amsterdam, the Netherlands, and his team have done just that, producing data hundreds of times more accurate than those obtained from previous experiments (see Nature doi:10.1038/news060417-7; 2006).

Using the Very Large Telescope in Paranal, Chile, they compared their results obtained with hydrogen in the lab with observations of light from two distant quasars. This light shines through clouds of hydrogen around 12 billion light years away. The lab result was smaller by $0.002 \%$ (E. Reinhold et al. Phys. Rev. Lett. 96, 151101; 2006).

A change of 20 parts per million over 12 billion years isn't large $-{ }^{\alpha}$ not jelly", as Andy
Fabian of the University of Cambridge, UK, puts it. But it could point to previously unknown subtleties in the way the Universe is put together. Such an effect is not explained by anything in physicists' standard model of particle physics.

The result has a confidence level of about 3.5-sigma, a statistical term that translates into a $0.3 \%$ possibility that it could be down to chance. That's good enough to be called an "indication", says Ubachs, but for such an important potential result it is not a cast-iron observation.

"You don't want to book a ticket to Stockholm on a 3.5-sigma result," chuckles John Webb, a physicist at the University of New South Wales in Sydney, Australia, who has also studied changes in the proton-electron mass ratio. "But they've done the best job of anyone so far on comparing the proton and electron mass."

The mass-ratio effect has until now received less attention than the fine-structure constant, $\alpha$, a measure of the electromagnetic force that keeps electrons in place inside atoms and molecules. Webb has been at the forefront of efforts to probe whether or not the mass ratio changes over time (J. K. Webb et al. Phys. Rev. Lett. 87, 091301; 2001). He expects to publish his most detailed study later this year, which relies on light from many more quasars than used in previous analyses.

But the work is controversial. Webb says he has been criticized by senior astrophysicists

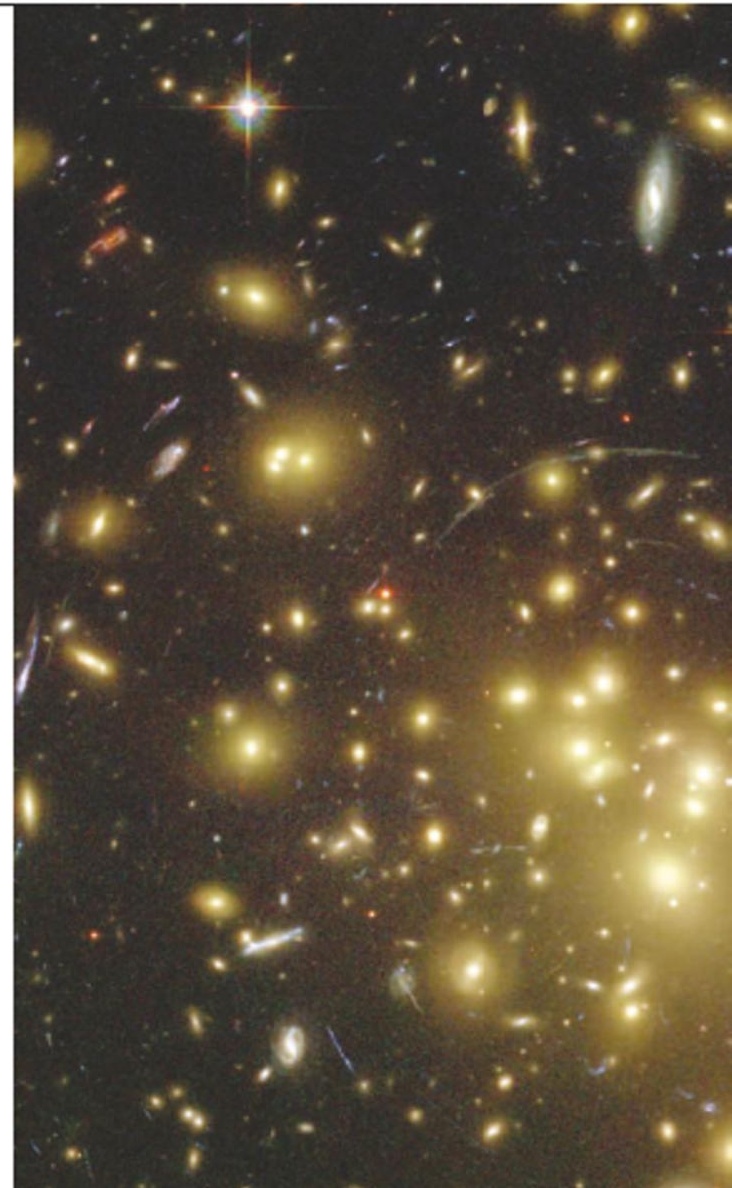

Weird stuff: observations of matter billions of light years away suggest the proton-electron . mass ratio has changed over eons.

for even tackling the problem. "It's as though § you're knocking a pillar of physics," he says.

John Barrow, a cosmologist at the University of Cambridge, adds that astronomers are often more sceptical than physicists simply because they are more aware of how complicated -

\title{
US particle physics fights for survival
}

The UnitedStates mustmake a bid to host the International Linear Collider (ILC), according to a report out this week from the National Academy of Sciences. If the multibillion-dollar particle accelerator is not builton US soil, the nation's high-energy physics community may be doomed.

High-energy physicists globally have endorsed the ILC as the next big project for the field. The collider,
30 kilometres in length, would smash together electrons and their anti-particles, known as positrons, at $500 \mathrm{GeV}$ in the hope of probing fundamental particles, including the yet-to-be-discovered Higgs particle.

"Without a serious bid, the community is going to atrophy," warns Harold Shapiro, an economist from Princeton University whochaired the panel that produced the report; the panel was convened by the US Department of Energy and the National Science Found ation, which together fund the lion's share of high-energy physics research in the United States. In addition torecommending that the government make a strong bid for the project, the panel suggests that it invests in high-energy astrophysics and neutrino research. There are currently around 2,000 high-energy physicists working in the United States.

The report comes at a precarious time for high-energy physics in America. Earlier this year, budget cutbacks forced the Relativistic Heavy lonCollider to take a private donation to continue operations, and the Tevatron, the nation's premier accelerator at the Fermi National AcceleratorLaboratory, or Fermilab, in Batavia, Illinois, is expected to 
